\title{
A 64 Gb/s PAM-4 Transimpedance Amplifier for Optical Links
}

\author{
Bart Moeneclaey, ${ }^{1}$ Jochen Verbrugghe, ${ }^{1}$ Elad Mentovich, ${ }^{2}$ Paraskevas Bakopoulos, ${ }^{3}$ \\ Johan Bauwelinck, ${ }^{1}$ and Xin Yin ${ }^{1}$ \\ ${ }^{I}$ Ghent University - imec, IDLab, Department of Information Technology, Technologiepark-Zwijnaarde 15, 9052 \\ Ghent, Belgium \\ ${ }^{2}$ Mellanox Technologies, Hakidma 26, Yokneam 2069200, Israel \\ ${ }^{3}$ National Technical University of Athens, School of Electrical \& Computer Engineering, Iroon Polytechniou 9 , \\ 15780 Athens, Greece \\ bart.moeneclaey@intec.ugent.be
}

\begin{abstract}
We present a $64 \mathrm{~Gb} / \mathrm{s}$ PAM-4 transimpedance amplifier with $180 \mathrm{~mW}$ power consumption. By switching between four gain modes, modulation amplitudes between -7 and at least $-0.2 \mathrm{dBm}$ yield a BER lower than $10^{-3}$.
\end{abstract}

OCIS codes: (200.4650) Optical interconnects; (060.2380) Fiber optics sources and detectors; (230.0250) Optoelectronics.

\section{Introduction}

The increasing popularity of multimedia applications drives an explosive growth in bandwidth requirements in datacenters. In order to meet these requirements, the IEEE has set up a task force aiming to define a $400 \mathrm{~Gb} / \mathrm{s}$ Ethernet specification. One of the solutions proposed by this task force entails 8 wavelength-multiplexed streams of $50 \mathrm{~Gb} / \mathrm{s}$ 4-level pulse-amplitude modulation (PAM-4) signals over single-mode fiber [1]. PAM-4 maps two bits to a symbol with normalized signal levels $+1,+1 / 3,-1 / 3$, or -1 . When compared to non-return-to-zero (NRZ), which is the traditional modulation format for these applications and maps only one bit to a symbol with normalized signal levels \pm 1 , PAM-4 thus allows twice the data throughput for a similar bandwidth. However, PAM-4 presents new challenges to the design of both transmitter and receiver, in particular the first stage of the receiver, i.e., the transimpedance amplifier (TIA). First of all, the use of multi-level modulation poses an increased linearity requirement on the amplifier. In order to maintain this linearity over a large range of optical input powers, some form of gain control is required. Moreover, as the minimal level spacing is now only one third of the NRZ level spacing, the sensitivity of the receiver is severely impaired. Finally, PAM-4 reception is more susceptible to the effects of intersymbol interference because, in the worst case, the interfering symbol is three times as large as the desired symbol [2].

This work introduces a TIA, developed for PAM-4 signaling up to $64 \mathrm{~Gb} / \mathrm{s}$ along single-mode links, avoiding the bandwidth-distance product limitation of multimode fiber and offering a viable solution when longer reach is required, e.g. in mega-datacenters [3] or in optically-switched flexible datacenter architectures [4]. We will first examine the architecture of the TIA, where we will discuss how the aforementioned challenges regarding PAM-4 reception have been dealt with. Next, experimental PAM-4 results are presented for $64 \mathrm{~Gb} / \mathrm{s}$ along with the measurement setup used to validate the TIA. Owing to the redesigned input stage and the use of gain control, we were able to greatly improve the TIA performance compared to our results presented in [5].

\section{Linear Receiver Architecture}

Figure 1(a) shows a simplified block diagram of the TIA, which was based on the TIA presented in [5], [6]. The datapath consists of a single-ended active-feedback TIA input stage, followed by a differential main amplifier and output stage. The balancing error integrator closes a feedback loop which removes the dc-offset between both output signals by adjusting the dc-voltage at the inverting input of the main amplifier. The feedback transconductance $g_{F}$ and the gain of the main amplifier can be digitally modified using the built-in controller, in order to keep the output signal swing and associated linearity at a reasonable level for varying optical input powers. In order to counteract the effect of a changing $g_{F}$ on the dynamic behaviour of the TIA input stage, the amplifier gain $A$ is adapted together with $g_{F}$ by adjusting its bias current and emitter degeneration. The TIA runs off a $2.5 \mathrm{~V}$ supply and draws $157 \mathrm{~mW}$ to $180 \mathrm{~mW}$, depending on the selected input stage bias current. A die, containing two identical TIAs, was manufactured in 

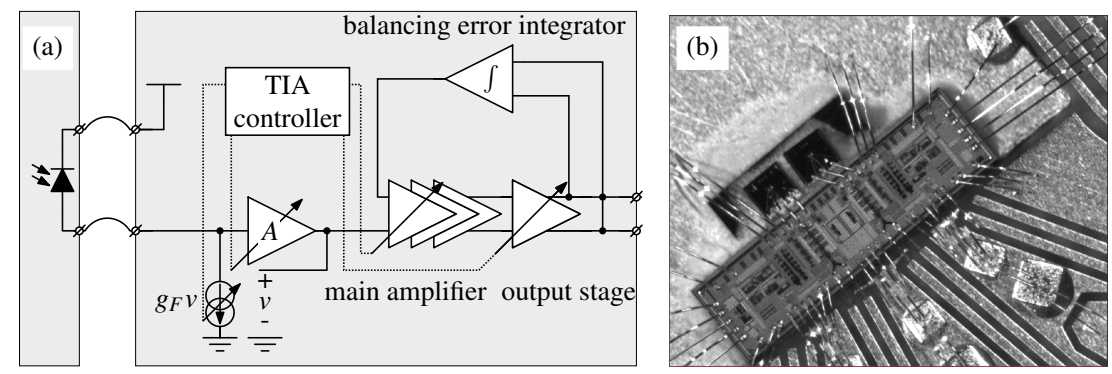

Fig. 1: (a) TIA block diagram and (b) die micrograph.

a $0.13 \mu \mathrm{m} \mathrm{SiGe} \mathrm{BiCMOS} \mathrm{process} \mathrm{and} \mathrm{is} \mathrm{shown} \mathrm{in} \mathrm{Fig.} \mathrm{1(b).} \mathrm{Each} \mathrm{TIA} \mathrm{occupies} 1500 \mu \mathrm{m} \times 890 \mu \mathrm{m}$ and was wirebonded to a PIN photodiode with a responsivity of $0.6 \mathrm{~A} / \mathrm{W}$.

\section{Experimental Setup}

The performance of the TIA was evaluated using bit-error rate (BER) measurements. The transmitter hardware is comprised of a continuous-wave $1550 \mathrm{~nm}$ laser and a $25 \mathrm{GHz}$ Mach-Zehnder Modulator, modulated by an electrical $64 \mathrm{~Gb} / \mathrm{s}$ PAM-4 signal yielding a $2.1 \mathrm{~dB}$ extinction ratio, i.e., the ratio between the optical powers corresponding to the normalized levels +1 and -1 . This signal is the result of Gray-mapping a $2^{9}-1$ pseudorandom binary sequence and is generated using a $64 \mathrm{GSa} / \mathrm{s}$ arbitrary waveform generator. At the receiving end, the optical signal is coupled into the photo diode by means of a manually aligned lensed fiber. The differential TIA output voltage is then captured at $160 \mathrm{GSa} / \mathrm{s}$ using a $63 \mathrm{GHz}$ real-time oscilloscope. The BER is obtained by offline symbol-by-symbol detection after the appropriate sample time and decision thresholds were determined. Similarly, eye diagrams and symbol histograms were generated offline based on the captured waveforms. The above procedure was repeated for varying input powers and four transimpedance gain modes, selected using the TIA's built-in controller. Note that, in order to accurately portray the performance of the TIA itself, no pre-emphasis or post-equalizer was used, as these would correct the TIA's frequency response and reduce intersymbol interference.

\section{Results and Discussion}

Figure 2(a) shows the measured BER curves for each of the four transimpedance gain modes versus the OMA, i.e., the difference between the optical power levels corresponding to the symbols +1 and -1 . Also shown in this figure is the BER curve resulting from choosing the appropriate gain mode at each point, i.e., the gain mode yielding the lowest BER, achieving the BER limit of $10^{-3}$ for OMAs between $-7 \mathrm{dBm}$ and at least $-0.2 \mathrm{dBm}$. Both the input-referred noise and the nonlinearity of the TIA are related to its transimpedance gain $R_{T}$, and are the dominant source of errors for respectively low and high OMAs. By reducing the transimpedance gain $R_{T}$, the BER curves are shifted toward higher OMAs. As such, the dynamic range is significantly improved by using gain control, selecting the appropriate gain mode at each point. For the points A-D, annotated in Fig. 2(a), eye diagrams were generated which are shown in Figs. 2(b) to 2(e), respectively, as well as symbol histograms and decision thresholds, shown in Figs. 2(f) to 2(i), respectively.

Compared to the $64 \mathrm{~Gb} / \mathrm{s}$ results from our previous contribution, [5], we were able to significantly improve the OMA sensitivity from $0 \mathrm{dBm}$ to $-7 \mathrm{dBm}$, as well as the dynamic range, with only a slight increase in power consumption from $165 \mathrm{~mW}$ to $180 \mathrm{~mW}$. Other state-of-the-art publications detailing PAM-4 BER measurements at similar bit rates report a higher TIA power consumption [7], [8] or no power consumption at all [9]. Real-time $60 \mathrm{~Gb} / \mathrm{s} \mathrm{BER}$ measurements are presented in [8], achieving a $B E R=10^{-3}$ sensitivity for an average optical input power of $-1.3 \mathrm{dBm}$ using a commercial TIA that consumes $1200 \mathrm{~mW}$. [7] and [9] present more complex experiments for which the TIA plays only a small role; their BER results are achieved using power-hungry digital signal processing techniques such as a feedforward equalizer and a decision-feedback equalizer. Furthermore, one of [7]'s results is achieved using an avalanche photodiode, whereas [9] used an Erbium-doped optical fiber amplifier with $25 \mathrm{~dB}$ gain in front of the photodiode to significantly boost the received input power. 

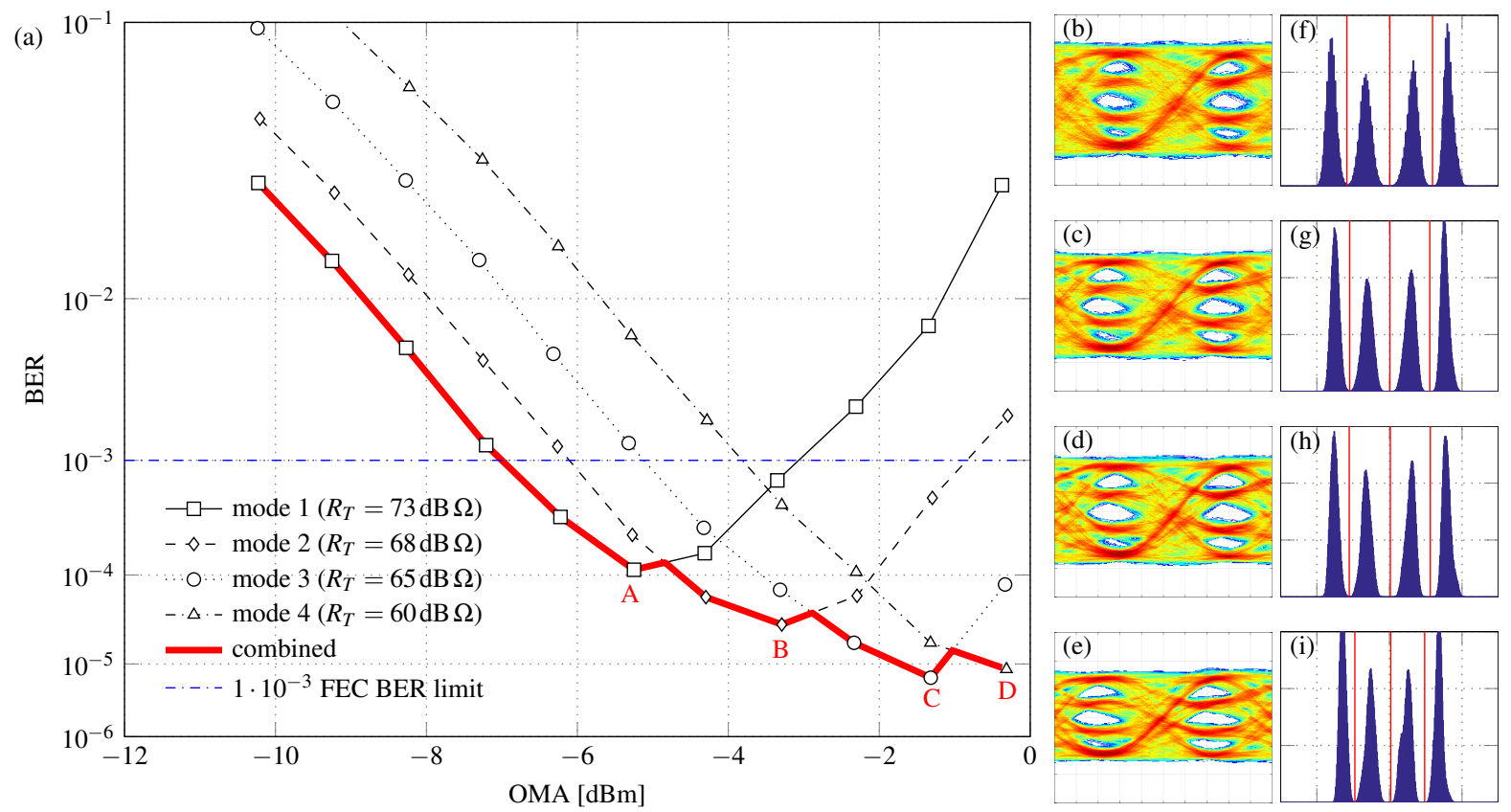

Fig. 2: (a) Measured BER versus OMA for $64 \mathrm{~Gb} / \mathrm{s}$ PAM-4, (b)-(e) eye diagrams for points A-D, respectively, and (f)-(i) symbol histograms for points A-D, respectively. Horizontal and vertical scale is $6.25 \mathrm{ps} / \mathrm{div}$ and $100 \mathrm{mV} / \mathrm{div}$ for all eye diagrams.

\section{Conclusion}

We have presented a TIA for optical links, implemented in $0.13 \mu \mathrm{m}$ SiGe BiCMOS and capable of $64 \mathrm{~Gb} / \mathrm{s}$ PAM-4 reception, for which it consumes $180 \mathrm{~mW}$. A built-in digital controller allows the TIA to switch between four discrete gain modes. The forward error correction BER limit of $10^{-3}$ is achieved for $64 \mathrm{~Gb} / \mathrm{s}$ for OMAs between $-7 \mathrm{dBm}$ and at least $-0.2 \mathrm{dBm}$.

\section{Acknowledgement}

This work was partially supported by the European Commision through the FP7 project MIRAGE (Contr. No. 318228) and the Institute for the Promotion of Innovation by Science and Technology in Flanders (IWT).

\section{References}

[1] IEEE P802.3bs baseline summary. [Online]. Available: http://www. ieee802.org/3/bs/baseline_3bs_0715.pdf (visited on 03/01/2015)

[2] J. Man et al., "A low-cost 100GE optical transceiver module for 2km SMF interconnect with PAM4 modulation," in Opt. Fiber Commun. Conf., 2014.

[3] D. Apostolopoulos et al., "Photonic integration enabling new multiplexing concepts in optical board-to-board and rack-to-rack interconnects," in SPIE OPTO, International Society for Optics and Photonics, 2014.

[4] N. Farrington et al., "A 10 us hybrid optical-circuit/electrical-packet network for datacenters," in Optical Fiber Communication Conference, Optical Society of America, 2013.

[5] B. Moeneclaey et al., "A 64 Gb/s PAM-4 linear optical receiver," in Opt. Fiber Commun. Conf., 2015.

[6] B. Moeneclaey et al., "A 40-Gb/s transimpedance amplifier for optical links," IEEE Photonics Technol. Lett., vol. 27, no. 13, pp. 1375-1378, Jul. 2015.

[7] Y. F. Chang, "Link performance investigation of industry first 100G PAM4 IC chipset with real-time DSP for data center connectivity," in Opt. Fiber Commun. Conf., 2016.

[8] P. Westbergh et al., "60 Gbits error-free 4-PAM operation with 850 nm VCSEL," Electron. Lett., vol. 49, no. 15, pp. 953-955, Jul. 2013.

[9] J. Wei et al., "Demonstration of the first real-time end-to-end 40-Gb/s PAM-4 for next-generation access applications using 10-Gb/s transmitter," J. Light. Technol., vol. 34, no. 7, pp. 1628-1635, 2016. 